\section{Differentialdiagnosen der Schizophrenie}

\author{
Jürgen Gallinat, Christiane Montag, Andreas Heinz
}

Psychiatrische Universitätsklinik der Charité im St. Hedwig-Krankenhaus Charité Universitätsmedizin Berlin

psychoneuro 2003; 30 (3): 146-152

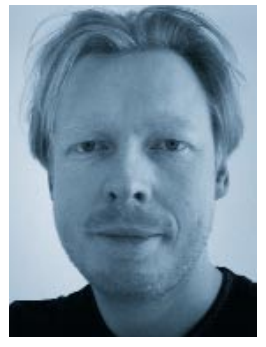

Jürgen Gallinat
Durch die operationalisierten Diagnosesysteme ICD-10 und DSM-IV ist die diagnostische Klassifikation der Schizophrenien international vereinheitlicht worden. Auf klinischer Ebene ergibt sich hieraus eine reliablere Diagnosestellung, während für die Erforschung schizophrener Erkrankungen eine bessere Vergleichbarkeit der Ergebnisse sowie mögliche Vorteile für die Lehre entstehen. Dieser Übersichtsartikel beschreibt die diagnostischen Kriterien sowie die häufigsten und wichtigsten Differentialdiagnosen der Schizophrenie.

D ie Diagnose der Schizophrenie wird heute in der Regel nach operationalisierten Kriterien gestellt in der Bestrebung, eine reliablere Schizophreniediagnostik zu gewährleisten. Die Diagnosesysteme ICD-10 und DSM-IV versuchen hierbei, die Erstrangsymptome Kurt Schneiders, die Grundsymptome Eugen Bleulers und die von Kraepelin betonte Bedeutung des Verlaufes zu kombinieren. In der ICD-10 sind im Kapitel F2 unter der Bezeichnung „Schizophrenie, schizotype und wahnhafte Störungen" relativ heterogene Störungen zusammengefasst. Im Gegensatz zu der DSM-Klassifikation werden in der ICD-10 auch schizotype Störungen, die in der DSM als Störungen der Persönlichkeit betrachtet werden, als eine Art Unterform der Schizophrenie aufgeführt, da sie zahlreiche für die Schizophre- nie charakteristische Symptome aufweisen und in Familienuntersuchungen bei schizophrenen Patienten gehäuft auftraten.

\section{Diagnose nach Erstrang- symptomen}

Die Diagnose Schizophrenie erfordert mindestens eines der nachfolgend aufgeführten Symptome, die sich an die Erstrangsymptome Kurt Schneiders anlehnen:

- Ich-Störungen: Gedankenlautwerden, Gedankenentzug, Gedankeneingebung, Gedankenausbreitung

- Kontrollwahn, Beeinflussungswahn, Gefühl des Gemachten (deutlich bezogen auf Körperbewegungen, bestimmte Gedanken oder Empfindungen), Wahnwahrnehmungen

- Kommentierende oder dialogisierende Stimmen
- Anhaltender kulturell unangemessener oder bizarrer Wahn.

Alternativ kann die Diagnose gestellt werden, wenn aus der folgenden Gruppe mindestens zwei Symptome bestehen:

- Anhaltende Halluzinationen jeder Sinnesmodalität, begleitet von undeutlich ausgeprägten Wahngedanken bei geringer Wahndynamik oder begleitet von anhaltenden überwertigen Ideen

- Gedankenabreißen oder Gedankeninterferenz (zeigt sich an Zerfahrenheit, Vorbeireden und Neologismen)

- Katatone Symptome wie Erregung, Haltungsstereotypien oder Flexibilitas cerea, Negativismus, Mutismus und Stupor

- Negativsymptome wie Apathie, Sprachverarmung, verflachter oder inadäquater Affekt, sozialer Rückzug.

Die Symptome dürfen nicht durch Substanzen oder organische Erkrankungen bedingt sein und müssen mindestens einen Monat andauern. Ausnahme ist die Schizophrenia simplex, für die eine Symp- 
tomdauer von mindestens einem Jahr gefordert wird. Von der Gruppe der Schizophrenien muss eine Reihe weiterer Erkrankungen, die psychotische Symptome verursachen können (symptomatische Psychosen), differentialdiagnostisch abgegrenzt werden.

\section{Symptomatische Psychosen}

Der Erkennung körperlicher Erkrankungen als Ursache für psychotische Symptome kommt große Bedeutung $\mathrm{zu}$, insbesondere in der Akutpsychiatrie und bei Erstmanifestation. Das Symptomprofil kann der Schizophrenie völlig gleichen, häufiger aber kommen optische Halluzinationen, Pseudohalluzinationen, Desorientiertheit und kognitive Beeinträchtigungen vor (10). So können Tumoren und zerebrale Traumen Positivsymptome (Wahn, Halluzination, Denkstörungen) induzieren - vor allem bei frontaler und temporaler Lokalisation - was sich mit pathophysiologischen Modellen der Schizophrenie gut vereinbaren lässt. Bei Lokalisation nahe dem limbischen System finden sich vor allem Angstsymptome (5). Epilepsien, vor allem komplex-fokale Anfälle mit temporalem Anfallsbeginn, können mit psychotischen Symptomen einhergehen, die iktal, postiktal und interiktal auftreten. Häufig finden sich akustische und olfaktorische Halluzinationen. Bei entzündlichen ZNS-Prozessen spielen Neurosyphilis, Neuroborelliose, Herpes-Enzephalitis und andere virale Enzephalitiden eine Rolle. Hierbei kommt es teils zu bizarren psychotischen Bildern mit Positivsymptomen und Fehlhandlungen. Die HIV-Enzephalopathie ist deutlich häufiger mit affektiven Störungen als mit psychotischen Symptomen vergesellschaftet. Auch zerebrovaskuläre Erkrankungen wie zerebrale Makro- und Mikroangiopathie, zerebrale Vaskulitis und Ischämien können von Positivsymptomen begleitet sein.

- Bei den Autoimmunerkrankungen wie dem systemischen Lupus erythematodes klagen die Patienten häufig über Depression und Müdigkeit, gelegentlich zeigen sich paranoid-halluzinatorische Syndrome
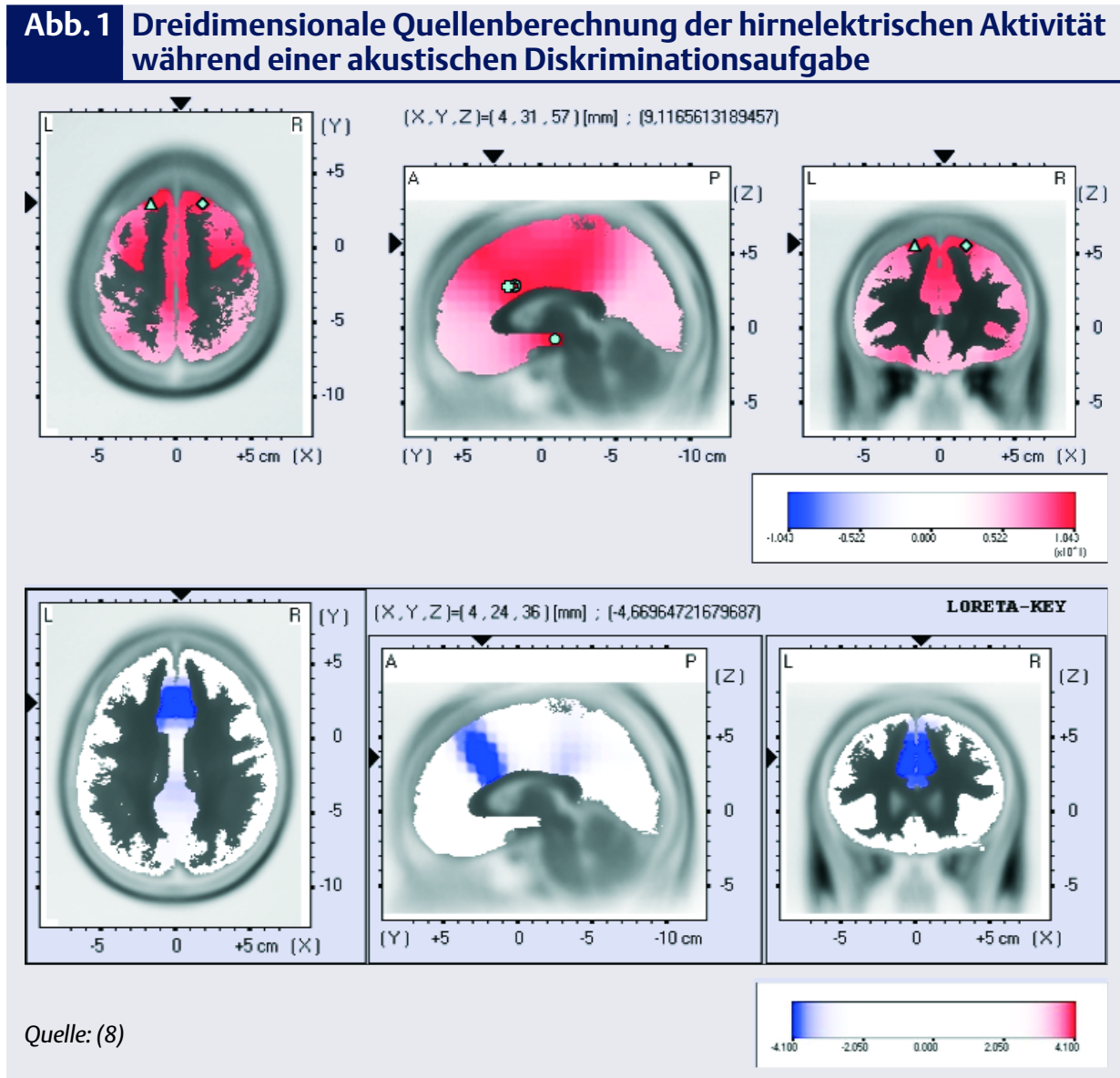

A) Bei Gesunden ist die Aktivität des akustischen Kortex und des anterioren Gyrus cinguli in rot dargestellt. B) Verglichen mit gesunden Probanden zeigen schizophrene Patienten eine verminderte Aktivität (blau) im anterioren Gyrus cinguli (kognitives Kompartiment) im Frontallappen. Das Defizit ist unter anderem Ausdruck von gestörten Aufmerksamkeits- und Monitoring Funktionen bei schizophrenen Patienten

- Die Multiple Sklerose geht häufig mit indifferent-euphorischer Stimmung einher, seltener kommt es zu psychotischen Symptomen

- Unter den Bewegungsstörungen ist für die Chorea Huntington typisch, dass im Frühstadium Persönlichkeitsveränderungen auftreten, die teilweise mit paranoiden Symptomen vergesellschaftet sind. Ähnliches findet sich bei metabolischen Störungen wie Hypoglykämie, Hyperglykämie, Morbus Wilson, Porphyrie, Morbus Cushing, Thyreotoxischer Krise und Vitamin- $\mathrm{B}_{12}{ }^{-}$ Mangel. In späteren Stadien der Chorea Huntington können schwere psychotische Symptome ausgebildet werden (15)

- Durch Medikamente und Medikamentenintoxikationen, vor al- lem durch Dopaminagonisten, Anticholinergika, Kortisol, Digitalis und Antibiotika können psychotische Symptome und Delirien auftreten.

\section{Substanzbedingte Psychose}

Durch den Konsum illegaler Drogen hervorgerufene psychotische Symptome stellen die häufigste Differentialdiagnose der Schizophrenie dar.

- Stimulanzien wie Amphetamine, Ecstasy, Ice (freebase) sowie Kokain können akut Antriebssteigerung, intensive Euphorie, Reizbarkeit, Wahnideen und Größenideen, bei Dauerkonsum auch depressive Episoden hervorrufen

- Die Halluzinogene LSD, Meskalin und Psilocybin erzeugen in der Intoxikation Positivsympto- 


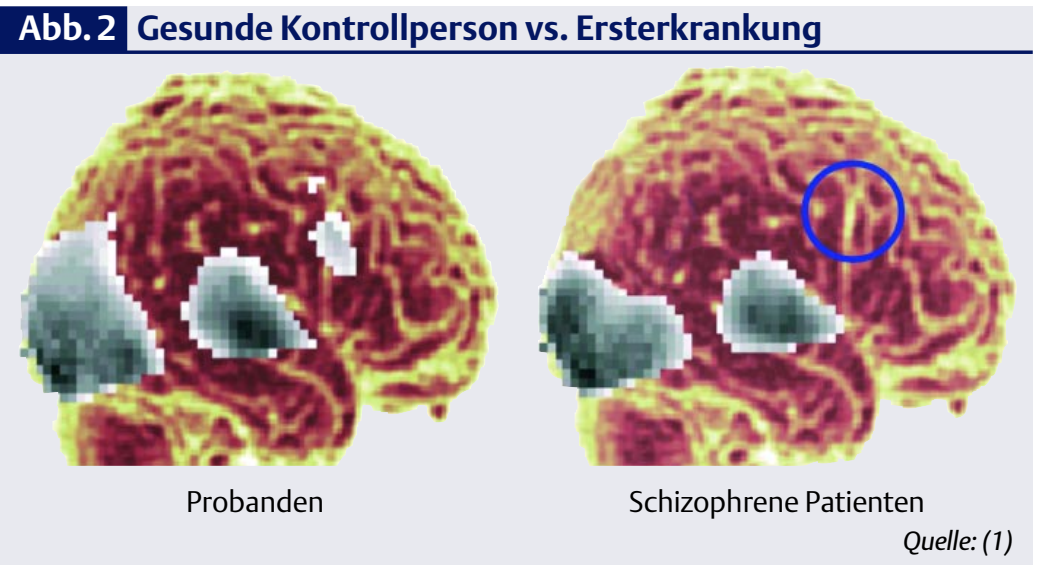

Normale und verminderte Aktivierung des dorsolateralen präfrontalen Kortex bei Präsentation komplexer visueller und akustischer Reizmuster. Eine verminderte Aktivierung findet sich bei schizophrenen Patienten, aber auch bei kortikalen Funktionsstörungen im Rahmen einer Alkoholabhängigkeit. Die Hirnregion ist ein wesentliches neurobiologisches Korrelat der Funktion des Arbeitsgedächtnisses, der Handlungsplanung und der Verhaltenskontrolle (siehe Artikel von Heinz und Juckel in diesem Heft)

matik. Auch erfahrene Konsumenten erleben Horrortrips, die typischerweise mit als real empfundenen Wahrnehmungsstörungen und Angst vor dem Verrücktwerden einhergehen. Anfällige Personen erleben prolongierte Psychosen. Flashbacks treten noch Monate nach dem letzten Konsum auf. Phencyclidin (PCP) besitzt eine hohe Potenz der Symptominduktion und induziert Positivsymptomatik, Antriebssteigerung mit aggressiven Durchbrüchen bis hin zu Selbstverstümmelungen. PCP blockiert Glutamat(NMDA)-Rezeptoren und erzeugt im Gegensatz zu anderen Substanzen auch Negativsymptomatik, so dass es für die biologische Ursachenforschung der Schizophrenie von besonderem Interesse ist. So zeigen Gesunde nach Einnahme von Substanzen dieser Stoffgruppe zerebrale Durchblutungsveränderungen (14) und kognitive Störungen wie bei schizophrenen Patienten (12). Im Gegensatz zu den USA wird die Substanz in Deutschland jedoch selten konsumiert

- Vor allem bei medizinischem Personal findet sich gelegentlicher Missbrauch des Anästhetikums Ketamin, welches ähnliche Symptome wie PCP indu- zieren kann. Der Gebrauch von Cannabis kann Depersonalisationserlebnisse und einen leicht ausgeprägten Verfolgungswahn induzieren. Seltener sind Halluzinationen. Bei hohen Dosen können prolongierte Cannabispsychosen auftreten, die bis zu sechs Wochen andauern. Mit höherer Wahrscheinlichkeit kommt es bei Personen mit einer Disposition für Schizophrenie zu einem Ausbruch der Symptomatik, welcher ohne Cannabiskonsum u.U. ausgeblieben wäre. Sicher belegt ist eine Verschlechterung des Krankheitsverlaufes schizophrener Patienten bei fortgesetztem Konsum. Problematisch ist, dass Patienten den akuten Rausch als angenehm erleben. Dies ist möglicherweise auf eine vorübergehende Reduktion von Negativsymptomen und Angst zurückzuführen

- Bei der akuten Intoxikation mit Alkohol sowie beim Entzugsdelir können zeitlich limitiert schizophrene Symptome vorkommen

- Die Symptomtrias Merkfähigkeitsstörungen, Desorientiertheit und Konfabulation bei der Wernicke-Korsakow-Enzephalopathie kann vordergründig an eine Schizophrenie denken lassen, auch wenn hier Desorien- tiertheit und Merkfähigkeitsstörungen nicht zu den charakteristischen Symptomen gehören (10)

- Bei der Alkoholhalluzinose zeigen sich ausgeprägte, meist akustische Halluzinationen in Form von Stimmenhören und typischerweise ein Verfolgungswahn mit Angst. Das Syndrom kann einer Schizophrenie stark ähneln, beginnt aber meist akuter und in höherem Lebensalter (40 bis 50 Jahre) bei relativ guter Prognose. Denkstörungen und Ich-Störungen sind sehr selten

- Bei Entzug von Benzodiazepinen kann es zu Überempfindlichkeit für sensorische Reize, veränderter Wahrnehmung und illusionärer Verkennung kommen. Das Auftreten von schizophrenen Symptomen ist selten.

\section{Psychische Störungen}

Die Diagnose einer akuten schizophreniformen psychotischen Störung ist zu stellen, wenn schizophrene Symptome kürzer als einen Monat bestehen. Dieses Zeitkriterium gilt auch, wenn die Remission durch Medikamente herbeigeführt wurde. Nosologisch ist dies etwas problematisch. Bei der wahnhaften Störung steht ein meist wenig bizarrer Wahn ohne Halluzinationen, formale Denkstörungen und Negativsymptomatik im Vordergrund. Letztgenannte Symptome bestehen aber nicht permanent und nur in geringer Intensität. Eine schizoaffektive Störung liegt vor, wenn affektive (depressive oder manische) und schizophrene Symptome gleichzeitig und innerhalb einer Krankheitsepisode über mindestens zwei Wochen (ICD10) bzw. einen Monat (DSM-IV) auftreten. Es ist zu beachten, dass bei der Schizophrenie Störungen der Affektivität im Sinne von Ängstlichkeit, Ambivalenz, inadäquatem Affekt und Parathymie häufig sind und nicht als schizoaffektiv zu werten sind. Bei wahnhaften Depressionen werden neben paranoiden Symptomen auch Halluzinationen beobachtet. Beide Symptome sind bei dieser Erkrankung synthym (stimmungskongruent), also thematisch der depressiven Stimmung entsprechend. Typisch 
sind Verarmungswahn, Schuldwahn, Versündigungswahn und Verkleinerungswahn. Bei akustischen Halluzinationen werden häufig vorwürfliche Stimmen angegeben. Die psychotischen Symptome verschwinden stets mit Abklingen der affektiven Symptome. Zwangsgedanken im Rahmen von Zwangserkrankungen werden in der Regel von Wahngedanken dadurch unterschieden, dass erstere vom Patienten als sinnlos erlebt werden und von einem inneren Widerstand begleitet sind. Bei genauerer Exploration zeigt sich allerdings nicht selten, dass viele Patienten diese Kriterien vermissen lassen, ohne dass eine Schizophrenie vorliegt. Epidemiologische Untersuchungen geben diskrete Hinweise dafür, dass Patienten mit Zwangsstörungen im Verlauf häufiger an Schizophrenie erkranken als die Normalbevölkerung (13), wobei die Datenlage für eine abschließende Beurteilung nicht ausreicht. Zwangsgedanken finden sich auch bei schizophrenen Patienten und zeigen häufig einen phasenhaften Verlauf. Antriebssteigerung, inhaltliche Denkstörungen (z.B. Größenwahn) sowie ausgeprägte assoziative Lockerung bei schweren Ma- nien können die Abgrenzung zur Schizophrenie erschweren. Neben dem Verlauf kann der synthyme Inhalt psychotischer Symptome als Kriterium herangezogen werden. Unter den Persönlichkeitsstörungen sind differentialdiagnostisch vor allem paranoide und schizoide Persönlichkeitsstörungen abzugrenzen. Hier kommen sozialer Rückzug, verminderte affektive Modulation und überwertige Ideen vor, in der Regel jedoch keine Positivsymptomatik. Außerdem bestehen Symptome von Persönlichkeitsstörungen über lange Zeit und zeigen keinen phasenhaften Verlauf (11). Die schizotype Störung zeigt ein exzentrisches oder seltsames Verhalten bei geringen sozialen Bezügen, magischem Denken, Misstrauen sowie ungewöhnlichen Wahrnehmungsinhalten. Es findet sich kein scharfer Beginn, und der Verlauf entspricht einer Persönlichkeitsstörung. Da keine klaren Grenzen zur Schizophrenia simplex sowie zur paranoiden- oder schizoiden Persönlichkeitsstörung bestehen, empfiehlt die ICD eine zurückhaltende Verwendung dieser Klassifikation. Dissoziative Störungen können mit bizarrem Verhalten einhergehen, welches im

\section{Abb. 3 Regionen gestörter zerebraler Aktivität bei depressiven Patienten}
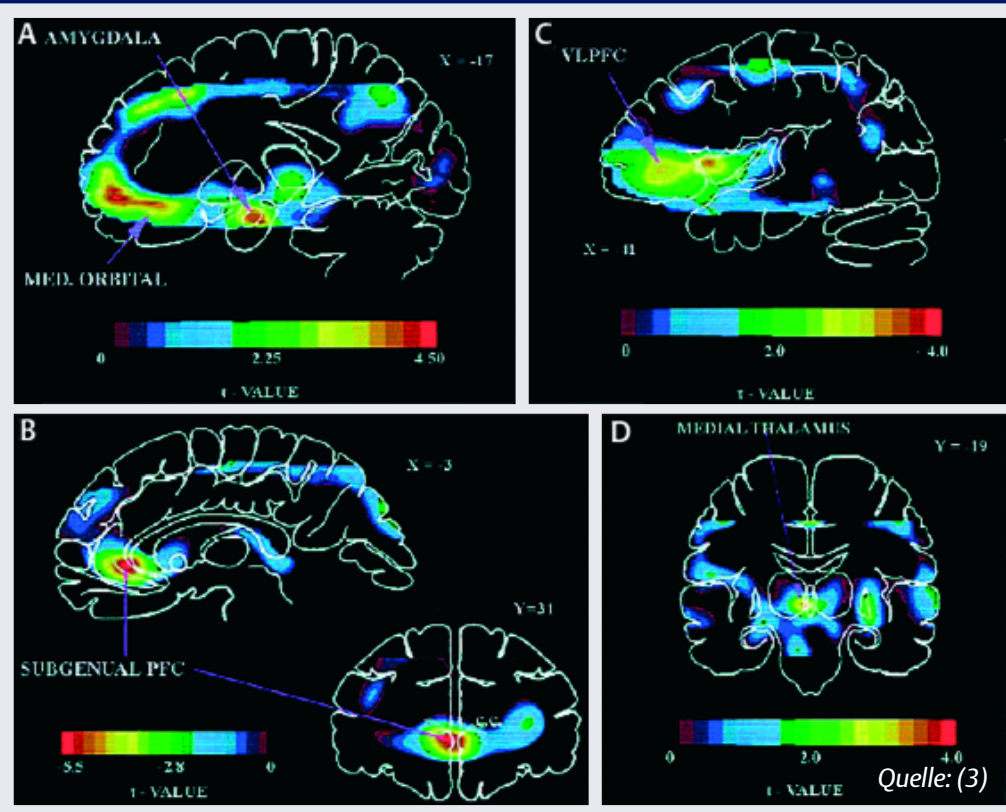

A) Amygdala und medialer orbitaler Kortex; B) subgenualer präfrontaler Kortex (emotionales Kompartiment des Gyrus cinguli); C) ventrolateraler präfrontaler Kortex und lateraler orbitaler Kortex; D) medialer Thalamus
Querschnitt der Schizophrenie ähneln kann. Das szenische Wiedererleben von traumatisierenden Ereignissen bei Borderline-Störungen, welches über Tage anhalten kann, kann als psychotisches Erleben fehlinterpretiert werden. Prinzipiell ist es möglich, bei einem Patienten die Diagnose Schizophrenie zusammen mit einer Persönlichkeitsstörung zu stellen.

\section{Apparative Untersuchungen in der Differentialdiagnostik}

Neben laborchemischen Untersuchungen und der Bildgebung steht vor allem das EEG zur Verfügung. Bei Patienten mit Schizophrenie wurden EEG-Veränderungen beschrieben, die jedoch zur Diagnostik nur bedingt geeignet sind (7). Auffällige Befunde bei organischen Störungen und Intoxikationen erlauben jedoch den Einsatz des EEG in der Differentialdiagnose. Eine Verlangsamung des Grundrhythmus und/oder eine Vermehrung von Theta- und Delta-Tätigkeit sind häufig bei Meningoenzephalitis, HIV-Enzephalopathie, Hypoglykämie, hyperglykämischen Koma, hepatischer Enzephalopathie, M. Cushing und anderen metabolischen Erkrankungen. Interessanterweise ist das EEG bei der Wernicke-Korsakow-Enzephalopathie und im Delirium tremens fast normal (überwiegend Beta Tätigkeit, „low-voltage EEG“). Bei anderen Delirformen sowie bei Intoxikationen mit Medikamenten oder Alkohol zeigt sich typischerweise eine Verlangsamung des Grundrhythmus und Delta-Theta Vermehrung. Auch postiktale Dämmerzustände zeigen ein verlangsamtes EEG, während das Bild bei postiktalen und interiktalen Psychosen uneinheitlich ist. Der generalisierte, nicht konvulsive Status epilepticus mit seiner teils bizarren Symptomatik kann durch spike-wave-Aktivität von der Schizophrenie unterschieden werden. Bei Verdacht auf Intoxikation, unklarem Fieber, Desorientiertheit und schwer einzuordnenden psychopathologischen Syndromen sollte daher ein EEG durchgeführt werden. Neuere Untersuchungen mittels dreidimensionaler Quellenanalyse von aufgaben-bezogener EEG-Aktivität zeigen bei schizophrenen Patienten Defizite vor allem im 
Frontallappen (Abb. 1), die hinsichtlich ihrer differentialdiagnostischen Wertigkeit derzeit überprüft werden (8).

\section{Forschungsergebnisse}

Zahlreiche bildgebende Untersuchungen, wie die funktionelle Magnetresonanztomographie (fMRT) und die Positronen-Emissions-Tomographie (PET), welche die zerebrale Aktivität über Veränderungen der Durchblutung darstellen, erlauben mittlerweile eine recht genaue Beschreibung gestörter Hirnfunktionen bei schizophrenen Patienten, haben bei psychischen Erkrankungen aber bisher allein wissenschaftliche Bedeutung. PET und fMRT zeigen bei schizophrenen Patienten im Vergleich zu Gesunden eine verminderte Aktivität im Frontallappen. Hier sind vor allem Regionen betroffen, die mit Kurzzeitund Arbeitsgedächtnis (dorsolateraler präfrontaler Kortex (1), Aufmerksamkeit, exekutiven Funktionen und Selbstmonitoring (kognitiver Anteil des anterioren Gyrus cinguli) sowie Sprache (Gyrus frontalis inferior) zu tun haben (Abb. 2). Darüber hinaus finden sich zahlreiche Berichte über gestörte Aktivität im mesialen Temporallappen, insbesondere in Hippokampus und parahippokampalem Gyrus, aber auch im temporalen Neokortex im Bereich der akustischen Hirnrinde. Interessanterweise finden sich Korrelationen zwischen temporalen Störungen und Positivsymptomen (4;8). Diese Beobachtungen lassen sich mit den lange postulierten Störungen der dopaminergen Neurotransmission bei Schizophrenie vereinbaren, da frühe Läsionen des medialen Temporallappens zu einer erhöhten oder überschießend stimulierbaren Dopaminfreisetzung führen können (9). Negativsymptomatik wurde häufig mit Veränderungen der Frontalhirnaktivität in Verbindung gebracht (2), die ihrerseits mit der dopaminergen Neurotransmission assoziiert ist (6).

Bei depressiven Erkrankungen findet sich ein anderes Profil zerebraler Funktionsstörungen (Abb. 3), das insbesondere Hirnregionen betrifft, die der Verarbeitung unangenehmer und bedrohlicher Reize dienen. Dazu gehört eine erhöhte Aktivität im me- dialen und lateralen orbitofrontalen Kortex, der bei emotionaler Verarbeitung und beispielsweise bei unlösbaren Aufgaben aktiviert wird, und die Amygdalae (Mandelkerne), die insbesondere durch bedrohliche Bilder stimuliert werden. Eine veränderte Aktivität zeigte sich auch im prägenualen und subgenualen präfrontalen Kortex (emotionaler Anteil des anterioren Gyrus cinguli) - einer prälimbischen Region, die an der affektiven Prozessierung, dem konditionierten Erlernen von Belohnungsreizen und der Motivationsbildung beteiligt ist (3). Diese Verfahren sind möglicherweise in Zukunft für die Diagnose und Differentialdiagnose der Schizophrenie verwendbar.

\section{Differential Diagnoses in Schizo- phrenia}

In the operationalised diagnostic systems ICD-10 and DSM-IV, the diagnostic classification of schizophrenia has been standardized internationally. At the clinical level, this has resulted in a more reliable establishment of the diagnosis, while in the field of research into schizophrenic diseases, the comparability of the results obtained has been improved; possibly, also, advantages in the area of teaching purposes have resulted. The present review article describes the diagnostic criteria and the most common and most important differential diagnoses of schizophrenia.

Key Words: schizophrenia - diagnosis - psychosis - illegal drugs

\section{Literatur}

1. Braus DF, Weber-Fahr W, Tost H, Ruf M, Henn FA. Sensory information processing in neuroleptic-naive first-episode schizophrenic patients: a functional magnetic resonance imaging study. Arch Gen Psychiatry 2002; 59: 696-701

2. Chua SE, Wright IC, Poline JB, Liddle PF, Murray RM, Frackowiak RS, Friston KJ, McGuire PK. Grey matter correlates of syndromes in schizophrenia. A semi-automated analysis of structural magnetic resonance images [see comments]. $\mathrm{Br}$ J Psychiatry 1997; 170: 406-410

3. Drevets WC. Neuroimaging studies of mood disorders. Biol Psychiatry 2000; 48: 813-829

4. Flaum M, O'Leary DS, Swayze VW, Miller DD, Arndt S, Andreasen NC. Symptom dimensions and brain morphology in schizophrenia and related psychotic disorders. J
Psychiatr Res 1995; 29: 261-276

5. Gallinat J, Hegerl U. Gesteigerte limbische Erregungsbildung bei Angstattacken. Der Nervenarzt 1998; 70: 206-215

6. Gallinat J, Bajbouj M, Sander T, Schlattmann P, Xu K, Ferro EF, Goldman D, Winterer G. Association of the G1947A COMT (Val(108/158)Met) gene polymorphism with prefrontal P300 during information processing. Biol Psychiatry 2003; 54: 40-48 7. Gallinat J, Hegerl U. Elektroenzephalographie in Neurophysiologische Untersuchungsmethoden in der Psychiatrie Edited by Hegerl U. Wien, Springer, 1998

8. Gallinat J, Mulert C, Bajbouj M, Herrmann WM, Schunter J, Senkowski D, Moukhtieva R, Kronfeldt D, Winterer G. Frontal and temporal dysfunction of auditory stimulus processing in schizophrenia. Neuroimage 2002; 17: 110-127

9. Heinz A, Bertolino A, Saunders RC, Kolachana BS, Jones DW, Gorey J, Lee KS, Knable MB, Weinberger DR. Dysfunction of prefrontal control of subcortical dopamine release in non-human promates with a neonatal mesial temporal lesion - implications for the developmental hypothesis in schizophrenia. Pharmacopsychiatry 1997; 177

10. Heinz A, Przuntek $H$, Winterer $G$, Pietzcker A. Clinical aspects and follow-up of dopamine-induced psychoses in continuous dopaminergic therapy and their implications for the dopamine hypothesis of schizophrenic symptoms. Nervenarzt 1995; 66: 662-669

11. Knutson B, Heinz A. Psychobiology of personality disorders in Textbook of biological psychiatry Edited by Panksepp J. Hoboken NJ, John Wiley \& Sons, 2003

12. Newcomer JW, Farber NB, Jevtovic-Todorovic V, Selke G, Melson AK, Hershey T, Craft S, Olney JW. Ketamine-induced NMDA receptor hypofunction as a model of memory impairment and psychosis. Neuropsychopharmacology 1999; 20: 106-118

13. Rasmussen SA, Eisen JL. Clinical and epidemiologic findings of significance to neuropharmacologic trials in OCD. Psychopharmacol Bull 1988; 24: 466-470

14. Tamminga CA, Thaker GK, Buchanan R, Kirkpatrick B, Alphs LD, Chase TN, Carpenter WT. Limbic system abnormalities identified in schizophrenia using positron emission tomography with fluorodeoxyglucose and neocortical alterations with deficit syndrome. Arch Gen Psychiatry 1992; 49: 522-530

15. Tost $H$, Wendt CS, Schmitt A, Heinz A, Braus DF. Huntington's disease: phenomenological diversity of a neuropsychiatric condition that challenges traditional concepts in neurology and psychiatry. Am J Psychiatry $2004 ; 161: 28-34$

\section{Korrespondenzadresse:}

Dr. Jürgen Gallinat

Psychiatrische Universitätsklinik der Charité im St. Hedwig-Krankenhaus

Charité Universitätsmedizin Berlin

Turmstraße 21

10559 Berlin

juergen.gallinat@charite.de 